\title{
Structure of the Majorana's equation and its physical interpretation
}

\author{
O.S. Kosmachev ${ }^{1, *}$ \\ ${ }^{1}$ VBLHEP, Joint Institute for Nuclear Research, Dubna, Russia
}

\begin{abstract}
It is generally believed that Majorana's paper [4] describes particles which have no antiparticles. A thorough analysis have shown that this paper gives rise to the questions the answers to which have not been realized up to now. By the Majorana equation we mean the equation that describes stable, massive, and neutral leptons. Fulfillment of these three requirements leads strictly and unambiguously to the conclusion that the Majorana equation describes a pair of stable, massive, and neutral leptons, a particle and an antiparticle. This is similar to the Dirac equation, but the spin properties of the leptons of this type differ from the spin properties of the electron or positron.
\end{abstract}

\section{Introduction}

While developing over time, relativistic nuclear physics (RNPh) as a problem of collisions of relativistic heavy ions has turned into a vast multifaceted project for in-depth study of physics of the microcosm. The project potentially contains all the currently known scope of particle physics. At the same time, it has revealed problems unsolvable within current theories and requires nontraditional approaches. The paper [1] is a convincing proof and illustration of well-established state. Among the important advances in the development of a new direction in the study of the microcosm there is a discovery of the cumulative effect and a quantitative description of the multiple particle production processes. These results primarily rely on the continuity concept and, as a consequence, on the hydrodynamics and thermodynamics methodology applied to multiple particle production problems.

At the same time, it is obvious that the phase of the process which is conventionally called formation of quark-gluon plasma, is an antithesis of continuity and a necessary condition to completely describe the collisions of relativistic nuclei. In terms of analysis of this structural aspect of the RNPh, noteworthy are the results recently obtained in the study of the leptonic sector.

A systematic group-theoretical analysis of the classical works [2], [3], [4] resulted in formulation of the Dirac algorithm (hereinafter referred to as the algorithm). It is a sequence of necessary operations to formulate lepton wave equations of the first order in space-time derivatives. As a result, we have obtained a holistic description of the lepton sector, which contains massive and massless, charged and neutral, stable and unstable leptons. All the obtained results are as rigorous as in the derivation of the Dirac equation. All lepton equations have been obtained without using the Lagrange method and fully comply with five initial assumptions. Physical completeness of the sector should be associated at least with the presence

\footnotetext{
*e-mail: kos@theor.jinr.ru
} 
of the entire range of observed leptons and then with a possibility of obtaining the maximum number of equations on the basis of the fixed assumptions. Internal self-consistency reflects similarity of leptons within the sector while preserving individual differences of each of the sector components. Note also closure of the description, which means that there are enough substructures to ensure individuality of each lepton and no redundancy.

In view of fundamentally of the fixed initial assumptions and completeness of the results obtained with the Dirac algorithm, questions arise as to how far we can go in applications of the Dirac algorithm and whether it can happen that the "building material" used for the lepton sector will be adequate for describing gauge bosons and hadrons. This material is the connected components of the irreducible representations of the Lorentz group. The irreducible representations are an infinite series of operators. Each member of the series can be written as one of four connected components. First, note that unstable leptons are a particular case of the positive answer to the above questions [5].

Asking these questions is motivated by the comprehensive and indisputable fact that all elementary particles eventually decay into stable leptons, photons, and almost stable protons. In this case, the monopoly of the so-called quark-gluon plasma as a unique suitable medium to form all leptons, photons, and hadrons in their finite variety becomes doubtful. Therefore, it is important to understand how complete and accurate our description of the lepton sector is.

\section{Holistic description of the lepton sector}

Below is a summary of some results related to the holistic description of the lepton sector [5]-[9].

The algorithm is based on five initial non-redundant assumptions.

1. Equations are invariant under the Lorentz group with allowance for four connected components and covariance in notation.

2. Formulation of the equations is based on the irreducible representations of the groups determining each lepton equation.

3. The four-vector probability current is conserved and the fourth current component is positively defined.

4. The lepton spin is taken to be $1 / 2$.

5. Each lepton equation of the first order in space-time derivatives must be reducible to the Klein-Fock-Gordon (KFG) equation that describes propagation of de Broglie waves for quantum particles.

Here is a summary of some results related to a holistic description of the lepton sector [5]-[9].

A not excessive and minimal set of initial requirements underlying Dirac algorithm is the following:

1. the equations must be invariant and covariant corresponding to the homogeneous Lorentz transformations taking into account all the four connected components;

2. the equations must be formulated on the basis of irreducible representations of the groups determining every lepton equation;

3. conservation of the four-vector probability current must be fulfilled and the fourth component of the current must be positively defined;

4. the lepton spin is supposed to be equal to $1 / 2$; 
5. every lepton equation must be reduced to the Klein-Fock-Gordon equation.

\section{The structural composition of the groups of stable leptons.}

- The group of Dirac equation $-D_{\gamma}(I I)\left\{\mathbf{d}_{\gamma}, \mathbf{b}_{\gamma}, \mathbf{f}_{\gamma}\right\}$,

- The group of Majorana equation for a doublet of massive neutrinos $-D_{\gamma}(I)\left\{\mathbf{d}_{\gamma}, \mathbf{c}_{\gamma}, \mathbf{f}_{\gamma}\right\}$,

- The group of Pauli equation for a quartet of massless neutrinos $-D_{\gamma}(I I I)\left\{\mathbf{d}_{\gamma}, \mathbf{b}_{\gamma}, \mathbf{c}_{\gamma}, \mathbf{f}_{\gamma}\right\}$,

- The group of equation for a massless $T$-singlet $-D_{\gamma}(I V)\left\{\mathbf{b}_{\gamma}\right\}$,

- The group of equation for a massless $(P T)$-singlet $-D_{\gamma}(V)\left\{\mathbf{c}_{\gamma}\right\}$,

Here $\mathbf{d}_{\gamma}$ is a subgroup of proper Lorentz representation.

All four subgroups $\mathbf{d}_{\gamma}, \mathbf{b}_{\gamma}, \mathbf{f}_{\gamma}, \mathbf{c}_{\gamma}$ are related by discrete transformations under the proper representation of the Lorentz group: time reversal $(\mathrm{T})$, space reflection $(\mathrm{P})$, and joint action of both transformations (PT): $\mathbf{b}_{\gamma}$ is a subgroup of $(T)$-conjugate representation, $\mathbf{f}_{\gamma}$ is a subgroup of $(P)$-conjugate representation, $\mathbf{c}_{\gamma}$ is a subgroup of $(P T)$-conjugate representation, i.e. $\mathbf{b}_{\gamma}=$ $(T) \mathbf{d}_{\gamma}, \mathbf{f}_{\gamma}=(P) \mathbf{d}_{\gamma}$, and $\mathbf{c}_{\gamma}=(P T) \mathbf{b}_{\gamma}$. Considering inverse discrete transformations, each of the four subgroups can be expressed in terms of any other subgroup.

It is evident from the above expressions that the connected components ensure structural individuality of the group of each lepton equation. Among them is a doublet (i.e., a particle and an antiparticle) of stable massive neutral particles. The group of this equation assumes that all matrices are reducible to the real form. We will call this equation (after some corrections) the Majorana equation. The group underlying the Majorana equation is designated as $D_{\gamma}(I)$.

Every group related with the corresponding equation has a nonrecurrent composition.

The structural composition of the groups for unstable leptons [5].

Group $\Delta_{1}$ has the following defining relations:

$$
\Gamma_{\mu} \Gamma_{v}+\Gamma_{v} \Gamma_{\mu}=2 \delta_{\mu v}, \quad(\mu, v=1,2,3,4,5)
$$

As a result we obtain the following composition: $\Delta_{1}\left\{D_{\gamma}(I I), D_{\gamma}(I I I), D_{\gamma}(I V)\right\}, \operatorname{In}\left[\Delta_{1}\right]=-1$.

Group $\Delta_{3}$ is defined by relations:

$$
\begin{array}{ll}
\Gamma_{s} \Gamma_{t}+\Gamma_{t} \Gamma_{s}=2 \delta_{s t}, & (s, t=1,2,3,4), \\
\Gamma_{s} \Gamma_{5}+\Gamma_{5} \Gamma_{s}=0, & (s=1,2,3,4), \\
\Gamma_{5}^{2}=-I . &
\end{array}
$$

It follows that the composition is: $\Delta_{3}\left\{D_{\gamma}(I I), D_{\gamma}(I), D_{\gamma}(I I I)\right\}, \quad \operatorname{In}\left[\Delta_{3}\right]=0$.

Group $\Delta_{2}$ is defined by relations:

$$
\begin{array}{ll}
\Gamma_{s} \Gamma_{t}+\Gamma_{t} \Gamma_{s}=2 \delta_{s t}, & (s, t=1,2,3), \\
\Gamma_{s} \Gamma_{4}+\Gamma_{4} \Gamma_{s}=0, & (s=1,2,3), \\
\Gamma_{4}^{2}=-I . & \\
\Gamma_{u} \Gamma_{5}+\Gamma_{5} \Gamma_{u}=0, & (u=1,2,3,4), \\
\Gamma_{5}^{2}=-I . &
\end{array}
$$

In this case we obtain the following:

$$
\Delta_{2}\left\{D_{\gamma}(I), D_{\gamma}(I I I), D_{\gamma}(V)\right\}, \quad \operatorname{In}\left[\Delta_{2}\right]=1 .
$$

All three groups have their own structures. We see that four conjugate components of Lorentz group allow different leptons to be described through complication of structural constituents. In curly braces, both for stable and unstable leptons, there are maximal invariant subgroups 
for the given group. It is on the basis of one of them that the expression for the probability current conservation is formulated.

Some consequences of the structure approach.

1. A possibility of listing all types of lepton equations within the framework of the initial assumptions is found.

2. Structural individuality of each lepton equation is defined.

3. The primary structural classification of leptons is formed.

4. A basis is laid for the relativistic description of unstable leptons. The structure of their equations indicates internal self-consistency of the entire lepton sector within the proposed algorithm.

5. Existence of an unstable massive neutrino is predicted.

6. Existence of "doubles" for $\tau^{ \pm}$-leptons, i.e., $\left(\tau^{*}\right)^{ \pm}$-leptons, is predicted. These four leptons make up a quartet state on the basis of a single group.

\section{Comments on the Majorana equations}

The structure of the Majorana equation like the structure of any other leptonic equation, completely determined by the group of the corresponding equation. In particular, the Dirac equation group is generated by known defining relations:

$$
\gamma_{\mu} \gamma_{v}+\gamma_{v} \gamma_{\mu}=2 \delta_{\mu \nu}, \quad \mu, v=1,2,3,4, \quad \gamma_{\mu}^{2}=1,
$$

Majorana made two mistakes in his work [4]. One of them could be corrected, and the second is unacceptably blunder. When transforming the Dirac equation, Majorana multiplies one of the Dirac group generators (one of four $\gamma$ - matrices in (4)) by the imaginary unit. It is easy to verify [12] that the Dirac group is thus transformed into a group of real matrices, and both groups do not go into each other under any similarity transformation. The dimension of the matrices of the irreducible representations remains the same $4 \times 4$, but it is senseless to apply matrices of one group to another group. Structural invariants of the Dirac and Majorana groups are different, $\operatorname{In}\left[D_{\gamma}(I I)\right]=-1$ and $\operatorname{In}\left[D_{\gamma}(I)\right]=1$. The defining relations for the Majorana group $D_{\gamma}(I)$ can be taken in the form:

$$
\begin{array}{ll}
\gamma_{s}^{\prime} \gamma_{t}^{\prime}+\gamma_{t}^{\prime} \gamma_{s}^{\prime}=2 \delta_{s t}, & \left(\gamma_{s, t}^{\prime}\right)^{2}=1 \quad(s, t=1,2,3) \\
\gamma_{s}^{\prime} \gamma_{4}^{\prime}+\gamma_{4}^{\prime} \gamma_{s}^{\prime}=0, & (s=1,2,3) \\
\left(\gamma_{4}^{\prime}\right)^{2}=-1 &
\end{array}
$$

It follows from relations (5) and the structural composition of the group $D_{\gamma}(I)$ ) that it is impossible to write the expression for probability current conservation on the basis of the subgroup $\mathbf{d}_{\gamma}$ because the group $D_{\gamma}(I)$ does not involve four anticommuting elements of the second order; otherwise, it would be the Dirac group. Expressions for the probability current conservation can be formulated on the basis of the subgroups $\mathbf{f}_{\gamma}$ and $\mathbf{c}_{\gamma}$. These groups are related by the (T)-transformation. It necessarily follows that the equation based on the group $D_{\gamma}(I)$ describes a particle and an antiparticle [9]. To find out the difference in the spin properties of the particles related to the Dirac and Majorana equations, we have used formulae for calculating weight numbers of irreducible representations of the Lorentz group [13].

In the case of the group $\mathbf{d}_{\gamma}$, the operators to be built are as follows:

$$
\begin{array}{ll}
H_{+}=i a_{1}-a_{2}, & F_{+}=i b_{1}-b_{2}, \\
H_{-}=i a_{1}+a_{2}, & F_{-}=i b_{1}+b_{2}, \\
H_{3}=i a_{3}, & F_{3}=i b_{3} .
\end{array}
$$


Here $b_{1}, b_{2}, b_{3}$ are the generators of the group $\mathbf{d}_{\gamma}, a_{1}, a_{2}, a_{3}$, are the generators of the subgroup of three-dimensional rotations in the group $\mathbf{d}_{\gamma}$. Weight numbers are eigenvalues of the operators $H_{3}=i a 3$ and $F_{3}=i b_{3}$. Calculation of the weight number for the operator $H_{3}$ for the subgroup $\mathbf{d}_{\gamma}$ yields $l_{0}=1 / 2$. In the case of the group $\mathbf{d}_{\gamma}$ any permutation of the operators $a 1, a 2, a 3$ in the formulae for $H_{+}, H_{-}, H_{3}$ does not change the value of $l_{0}$ because all three operators $a 1, a 2, a 3$ as elements of the group $\mathbf{d}_{\gamma}$ are all of the fourth order. The situation is different in the group $D_{\gamma}(I)$, where the similar operators have to be built on the basis of the subgroup $\mathbf{f}_{\gamma}$. The structure of the group $\mathbf{f}_{\gamma}$ assumes that only one of its three elements $a_{1}, a_{2}, a_{3}$, is anti-Hermitian. Therefore, real eigenvalues $l_{0}=1 / 2$ of the operator $H_{3}$ appear when action is exerted on one of the three elements. In two other cases, imaginary values arise. This means selection of the spin quantization axis. The selected direction can only be the particle momentum direction. Thus, the Majorana equation describes doublet states of leptons, i.e., particles and antiparticles. The particle spin can be directed along or opposite the momentum.

As for the unacceptable error mentioned above, it could not be unnoticed before. To verify this, refer to the texts:

- Ettore Majorana, [4] (in original Italian)

"Poniamo $\psi=U+i V$ e consideriamo le equazioni reali (8') in quanto agiscone sulle $U . . . "$ Il vantaggio di questo procedimento rispetto all' interpretazione elementare delle equazioni di Dirac è (come vedremo meglio fra poco) che "non wi è più nessuna ragione di presumere l'esisteza di antineutroni o antineutrini.

- Condon E. and Shortley G., [11]

"The distinction between $\Psi$ and $\bar{\Psi}$ is more fundamental than that between ordinary complex conjugates; there is no sense in which we can split $\Psi$ into a real and an imaginary part."

- Dirac P., [10].

"Our bra and ket vectors are complex quantities, since they can be multiplied by complex quantities and are then of the same nature as before, but they are complex quantities of special kind which cannot be split up into real and pure imaginary parts."

Majorana's idea to split the solution to the Dirac equation into the real and imaginary parts is actually the abandonment of the concept of the Hilbert space without anything offered in exchange. Majorana's conclusion that the neutron as he constructed it could not have an antiparticle turned out to be erroneous. At the same time, it is known that there exist true neutral particles, photons and neutral pi mesons. As to our description of the lepton sector, it includes leptons which have no antiparticles. These are particles relating to the groups $D_{\gamma}(I V)$ and $D_{\gamma}(V)$, but they are massless.

\section{Conclusion}

Dirac's algorithm has been formulated on the basis of structural analysis groups of lepton equations known for a long time [2], — [4]. The most significant characteristic of the algorithm is that the structural elements of the groups turned out to be four connected components of the Lorentz group. For this reason, there are no principal prohibitions to generalize the algorithm to a more complex objects such as quarks and hadrons. 


\section{References}

[1] A.M. Baldin, A.A. Baldin, Phys.Particles and Nucl. 29(3), 577-630 (1998)

[2] P. Dirac, Proc.Roy. Soc. A, 117, 610-624 (1928)

[3] W. Pauli, Handbuch der Physik (Springer, Berlin, 1933) 226

[4] E. Majorana, Il Nuovo Cimento. 14, 171-184 (1937)

[5] A.A. Gusev, O.S. Kosmachev, Phys. Part. Nucl. Lett. 5, 67-71 (2008)

[6] O. Kosmachev, Phys. Part. Nucl. Lett. 7, 149-174 (2010)

[7] O. Kosmachev, Phys. of At. Nucl. 74(6), 930-935 (2011)

[8] O. Kosmachev, Phys. of At. Nucl. 75(7), 885-887 (2012)

[9] O. Kosmachev, JINR Preprint P4-2017-97 (2017) (in Russian)

[10] P. Dirac, The principles of Quantum Mechanics (Oxford, 1958)

[11] E. Condon and G. Shortly The theory of atomic spectra (Cambridge, 1959)

[12] J. Lomont Applications of Finite Groups (Academic Press, London, New York, 1959)

[13] I. Gelfand et.al. Representations of rotation group and Lorentz group, their applications (Phis-Mat. Press, Moscow, 1958) (in Russian) 